\title{
RESEÑA
}

\section{ROSALES, Osvaldo (2020). El sueño chino. Cómo se ve China a sí misma y cómo nos equivocamos los occidentales al interpretarla. Siglo XXI Editores Argentina, Buenos Aires, Argentina}

"El sueño chino - Cómo se ve China a sí misma y cómo nos equivocamos los occidentales al interpretarla", cuenta con el prólogo escrito por Alicia Bárcena, Secretaria Ejecutiva de la CEPAL, una introducción y 13 capítulos. El autor del libro es el economista chileno Osvaldo Rosales, quien fue Asesor Regional y Director de la División de Comercio Internacional e Integración de la CEPAL y también se desempeñó como Director de Relaciones Económicas Internacionales en la Cancillería de su país.

En su obra Rosales expone los prejuicios de occidente con relación a China, dando a conocer la otra cara de la moneda a partir de una visión que hace eje en la propia China. Para ello, realiza un recorrido a lo largo de su historia desde comienzos de la dinastía Ming a mediados del siglo XIV cuando era el país más avanzado del mundo en materia económica y científica, hasta nuestros días. El actual presidente Xi Jinping, quien hizo suya la noción del "sueño chino", en realidad recupera la idea de restituir a la normalidad histórica al País del Centro.

En la introducción el autor presenta las distintas temáticas que se desarrollan en el libro haciendo énfasis en el "sueño chino", que según Rosales se inicia a partir de Deng Xiaoping con la reforma y apertura y continúa con todos los líderes que le sucedieron en el Partido Comunista Chino, aunque el concepto de "sueño chino" como tal fue explicitado en la etapa de Xi Jinping. En la visión del autor, la noción "sueño chino" se debe entender como la reconquista del poderío e influencia que el país tenía en el siglo XV. Actualmente, va de la mano de otro concepto clave, el "Made in China 2025", cuyo objetivo es hacer de China una potencia tecnológica líder en innovación a largo plazo. Para ello, el gobierno propone reducir la brecha tecnológica con los países líderes hacia 2025 y alcanzar la primacía en innovación global en 2045. El autor explica que China fue cumpliendo etapas intermedias vinculadas con la educación, el progreso económico, ambiental y social con la finalidad de lograr esos objetivos.

En el capítulo 1 Rosales analiza en profundidad el "sueño chino" promovido por Xi Jinping, considerándolo la síntesis de los ideales planteados por otros tres grandes líderes chinos como Sun Yat-sen, Mao Zedong y Deng Xiaping. Según el autor, éste significa el retorno al estado previo al Siglo de Humillación y la recuperación de la normalidad histórica con China como epicentro de la economía global. Este nuevo camino comenzó el 1 de octubre de 1949 con el triunfo del Ejército Popular de Liberación y la constitución de la República Popular China.

El capítulo 2 se focaliza en el surgimiento de la República Popular China, narrando distintas vicisitudes vinculadas al desgaste de la dinastía Qing, contexto en el 
cual se crearon movimientos a favor de la República. En este marco, se centra en la historia y en el pensamiento del primer presidente de la República, proclamado padre de la misma, Sun Yat-sen, quien veía en el proteccionismo del Imperio una de las primeras causas de la decadencia China.

En el capítulo 3 se desarrolla el período del líder revolucionario Mao Zedong y sus políticas, con especial atención al Gran Salto Adelante (1958-1961) y a la Revolución Cultural Proletaria (RCP) (1966-1969/1970). Rosales analiza sus políticas económicas, que pusieron énfasis en la industria y en la agricultura intensiva, y su política exterior, puntualmente la ruptura con la entonces Unión Soviética (URSS).

El capítulo 4 aborda la etapa de Deng Xiaoping como máximo líder del país. Su gobierno buscó retornar a la idea de Mao de que a las distintas circunstancias se deben aplicar diferentes medidas con la intención de justificar el cambio de rumbo en materia de política económica y aplacar las críticas de sus disidentes. Bajo su mandato se buscó alcanzar el desarrollo de las economías occidentales más avanzadas, mejorar las condiciones sociales de vida y apostar al crecimiento económico y a la estabilidad del partido para salvaguardar al socialismo. En esta etapa surgió el conocido "socialismo con características chinas".

Siguiendo con el análisis del gobierno de Deng, en el capítulo 5 Rosales explica cómo en su período se vivió un gran crecimiento económico y una triple transición, transformando el país de una economía cerrada a una abierta, de una planificada a una de mercado y de una sociedad rural a una urbana. Estas transformaciones se explican en las reformas agrícolas, urbanas y en la creación de las Zonas Económicas Especiales. Deng inició el camino para que la tercera y cuarta generación de líderes profundizaran la apertura. Un ejemplo de ello fue el inicio de negociaciones para la reincorporación a la Organización Mundial del Comercio (OMC) iniciada en el gobierno de Deng y alcanzada en 2001 bajo la presidencia de Jiang Zemin.

El capítulo 6 desarrolla el gobierno de Xi Jinping como exponente de la quinta generación de líderes, siendo el primer presidente nacido luego de la proclamación de la República Popular. Xi recoge los principios de los líderes que lo precedieron, persiguiendo la gran revitalización china postulada por primera vez por Sun Yat-Sen. Por otra parte, retoma las directrices de Deng en torno a la reforma y apertura, la centralidad del desarrollo económico y en la innovación tecnológica y científica con el programa "Made in China 2025". Rosales remarca cómo esta mirada a largo plazo diferencia a los líderes chinos con respecto a los occidentales. Otras medidas que caracterizan el gobierno de Xi son la Iniciativa de la Franja y La Ruta (OBOR), el Banco Asiático de Inversión en Infraestructura (AIIB) y el Nuevo Banco de Desarrollo del BRICS. El sello de discontinuidad con respecto a sus antecesores lo encuentra en la lucha contra la corrupción, en la demostración externa del poderío real de China y en la vuelta al culto del líder que con Deng se había intentado desarraigar.

En el capítulo 7 el autor analiza los desafíos en clave económica que ha de afrontar China. Con relación a ello, Rosarles menciona como temas más importantes la sostenibilidad del crecimiento con base en el consumo, el fortalecimiento del Renminbi como moneda internacionalizada, la sobreproducción de su tejido industrial y su reconversión productiva, el cuidado ambiental, el empleo y el envejecimiento de la 
población, la deuda privada y las burbujas inmobiliarias y la brecha de ingresos entre distintos sectores de la sociedad.

En el capítulo 8 se expone la distancia existente entre la perspectiva occidental y la realidad en cuanto a la existencia y a la intensidad del debate político al interior de China. Un dato relevante que brinda el autor es que existen en el seno del Partido Comunista Chino al menos otras dos corrientes distintas de pensamiento bien definidas con respecto al manejo de la economía, con los neoconservadores por un lado y la neoizquierda por el otro. Los neoconservadores creen que el modelo de crecimiento económico se está agotando y debe girar hacia uno donde los emprendedores tengan más libertades para poder seguir innovando. La neoizquierda en cambio valora la apertura y el mercado, pero dándole prioridad a la democracia y la igualdad sobre el mercado mismo.

Desde el capítulo 9 Rosales se centra en la relación conflictiva entre China y Estados Unidos. El autor presenta los rasgos generales de la disputa y cómo China busca evitarla para mantener la estabilidad global y así poder alcanzar el "sueño chino". En los apartados siguientes del capítulo presenta los alegatos estadounidenses contra China, por ejemplo: la sobreoferta y los subsidios a la industria china, la baja observancia de los derechos de propiedad intelectual, las compras públicas y la apertura en el sector financiero. De la misma manera, presenta los argumentos chinos fundados en el trato discriminatorio recibido por parte de Estados Unidos y de la Unión Europea en el seno de la OMC, las acusaciones sobre manipulación cambiaria, el bloqueo al acceso tecnológico estadounidense y la prohibición a la inversión china en empresas de dichos países.

En el capítulo 10 el autor avanza sobre los escenarios posibles con respecto al desarrollo de la "guerra comercial". Entiende que tras su velo hay una especie de "guerra larvada" o conflicto que no debe ser subestimado. En función de esta apreciación, Rosales examina el conflicto en clave del libro El Arte de la Guerra analizando aspectos como el poder del líder y el momento histórico del conflicto para determinar cuál de los oponentes se encuentra en una mejor posición.

El capítulo 11 desarrolla la competencia por la hegemonía tecnológica entre China y Estados Unidos. La raíz de la misma se encuentra en el creciente peso de China en las ciencias mundiales y su aumento del gasto en la innovación para disputar el liderazgo para 2050. El autor considera que en el momento actual del conflicto no está aún definido a favor de quien se terminará inclinando la balanza.

En el capítulo 12 el autor compara la guerra comercial y tecnológica entre China y Estados Unidos con la guerra fría entre Estados Unidos y la ex URSS, marcando las diferencias. En primer lugar, no es pertinente comparar el desarrollo tecnológico y económico de la China actual con la URSS de la guerra fría, en tanto China es mucho más avanzada. En segundo lugar, a diferencia de la ex URSS, China se encuentra integrada en el tejido comercial mundial. De acuerdo con Rosales, el conflicto actual no puede reducirse a lo comercial dado que hasta la dimensión militar está incluida en la conflictividad en virtud de que los avances tecnológicos impactan en esa dimensión. En este capítulo se plantea cuál sería el mejor sistema político de adoptar, comparando costos y beneficios del modelo occidental y el llamado "mandarinato chino". 
El capítulo 13 evalúa los márgenes de acción de América Latina para evitar caer en la lógica del conflicto entre China y Estados Unidos. En ese sentido, plantea la viabilidad de la opción multilateral y propone estrategias destinadas a evitar caer en opciones entre uno u otro de los dos países en disputa. Además, explica cómo muchos líderes occidentales se equivocaron en pensar que China con toda su historia pudiera sucumbir y aceptar el modelo político occidental basado en la democracia liberal.

La obra de Osvaldo Rosales muestra con claridad cómo se proyecta el "sueño chino" dando una descripción detallada de las principales características de la historia política y económica de China de los últimos 70 años. A través de su mirada, el autor despeja todas las dudas y los prejuicios sobre el país, la unidad del partido y de sus dirigentes. Asimismo, su libro aporta conocimientos y consideraciones sobre el rol de China en innovación científica y tecnológica en el nuevo orden internacional.

Por Emanuel Follis y Carolina Silva - Miembros del Grupo de Estudios sobre China y Argentina (GEChinA). Facultad de Ciencia Política y Relaciones Internacionales. Universidad Nacional de Rosario (UNR). Correos electrónicos: follisemanuel@gmail.com y scarolina342@gmail.com ORCID: 0000-0003-4473-2089 y 0000-0003-2584-8449. 